\title{
The infrared imaging spectrograph (IRIS) for TMT: status report for IRIS imager
}

Yutaka Hayano, Ryuji Suzuki, Mizuho Uchiyama, Fumihiro Uraguchi, Bungo Ikenoue, et al.

Yutaka Hayano, Ryuji Suzuki, Mizuho Uchiyama, Fumihiro Uraguchi, Bungo Ikenoue, Yoshiyuki Obuchi, Sakae Saito, Yoko Tanaka, Takashi Nakamoto, Koji Nakamura, James E. Larkin, Shelley A. Wright, Eric M. Chisholm, Jennifer S. Dunn, Robert W. Weber, David Andersen, "The infrared imaging spectrograph (IRIS) for TMT: status report for IRIS imager," Proc. SPIE 10702, Ground-based and Airborne Instrumentation for Astronomy VII, 10702A8 (29 July 2018); doi: 10.1117/12.2314327

EDent: SPIE Astronomical Telescopes + Instrumentation, 2018, Austin, Texas, United States 


\title{
The infrared imaging spectrograph (IRIS) for TMT: Status report for IRIS imager
}

\author{
Yutaka Hayano*a, Ryuji Suzuki ${ }^{\text {a }}$, Mizuho Uchiyama ${ }^{\mathrm{a}}$, Fumihiro Uraguchi ${ }^{\mathrm{a}}$, Bungo Ikenoue ${ }^{\mathrm{a}}$, \\ Yoshiyuki Obuchi ${ }^{a}$, Sakae Saito ${ }^{a}$, Yoko Tanakab ${ }^{b}$ Takashi Nakamoto ${ }^{a}$, Koji Nakamura ${ }^{a}$, James E. \\ Larkin $^{\mathrm{c}}$, Shelley A. Wright ${ }^{\mathrm{d}}$, Eric M. Chisholm ${ }^{\mathrm{e}}$, Jennifer S. Dunn ${ }^{\mathrm{f}}$, Robert W. Weber ${ }^{\mathrm{g}}$, David \\ Andersen $^{\mathrm{f}}$

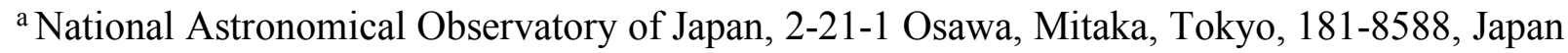 \\ a Subaru Telescope, National Astronomical Observatory of Japan, 650 North A'ohoku Place, Hilo HI \\ 96720, USA \\ ${ }^{c}$ Department of Physics and Astronomy, Univ. of California, Los Angeles, CA 90095-1547, USA \\ ${ }^{d}$ Center for Astrophysics and Space Sciences, Univ. of California, San Diego, La Jolla, CA 92093, \\ USA \\ e Thirty Meter Telescope International Observatory, 100 W Walnut St, \#300, Pasadena, CA 91124, \\ USA \\ ${ }_{\mathrm{f}}$ National Research Council of Canada - Herzberg, $5071 \mathrm{~W}$ Saanich Rd, Victoria, V9E 2E7, Canada \\ ${ }^{\mathrm{g}}$ Caltech Optical Observatories, 1200 E California Blvd., Pasadena, CA 91125, USA
}

\begin{abstract}
The current status of IRIS imager at NAOJ is reported. IRIS (Infrared Imaging Spectrograph) is a first light instrument of TMT (Thirty Meter Telescope). IRIS has just passed the preliminary design review and moved forward to the final design phase. In this paper, optical and mechanical design of IRIS imager and prototyping activities conducted during the preliminary design phase are summarized.
\end{abstract}

Keywords: Extremely Large Telescope, TMT, Infrared Instrument, IRIS

\section{INTRODUCTION}

\subsection{IRIS overview}

The Infrared Imaging Spectrograph (IRIS) ${ }^{1}$ is a cryogenic instrument under development for first-light operation of the Thirty Meter Telescope (TMT). IRIS combines a "wide-field" imager with an integral field spectrograph (IFS) and covers the $0.84 \mu \mathrm{m}$ to $2.4 \mu \mathrm{m}$ wavelength range. IRIS is an Adaptive Optics (AO) assisted instrument, and receives its optical input from the TMT facility laser guide star multi-conjugate AO (MCAO) system NFIRAOS ${ }^{2}$.

The IRIS imager has a total field-of-view (FoV) of $34 \times 34$ arcsec at a plate scale of 4 milliarcseconds (mas). The IFS offers four spaxel scales ranging from 4 mas to 50 mas, and is capable of generating up to 14,000 simultaneous spectra within a filled rectangular pattern. The imager and IFS are encased by the science cryostat. Furthermore, three oninstrument wavefront sensors (OIWFS) are mounted in a separate enclosure atop the science cryostat, cooled by a liquid nitrogen. OIWFS patrol the exterior perimeter of the two arc minute diameter field and provide measurements of the tip/tilt, focus (TTF) and plate scale modes. IRIS also mates to NFIRAOS through a support structure. The science cryostat further connects to a rotator, which enables IRIS to provide its own field de-rotation. IRIS cable wrap routes the cables from the inside the OIWFS and science cryostat to the Nasmyth platform below the instrument.

*hayano@naoj.org; phone +81 42234 3941; fax +81 422343865

Ground-based and Airborne Instrumentation for Astronomy VII, edited by Christopher J. Evans, Luc Simard, Hideki Takami, Proc. of SPIE Vol. 10702, 10702A8 · ( 2018 SPIE · CCC code: 0277-786X/18/\$18 · doi: 10.1117/12.2314327 


\subsection{Development phase of IRIS}

Based on the guideline of the TMT project, IRIS is developed through a sequential phase strategy. In 2005 , the development of IRIS started with the feasibility study phase, followed by the conceptual design phase which completed in 2011. The preliminary design phase was then initiated upon the completed review of the conceptual design. This preliminary design phase consisted of two stages with the focus placed on preliminary opto-mechanical designs for the first stage, and on software, electronics, control system and system engineering and management for the second stage. The first major review for the opto-mechanical designs was held in November 2016, and the second major review was conducted in September 2017.

\section{OPTICAL LAYOUT AND PACKAGING}

The science optical path of IRIS starts from imager optical train and relays the optical beam to the IFS just prior to the imager detectors by pickoff mirrors. This optical design is termed as "sequential design".

The IRIS imager provides diffraction limited imaging performance in the wavelength range of 0.84 to $2.4 \mu \mathrm{m}$. It covers a 34" x 34" FOV with 4 mas spatial sampling and uses four Hawai 'i-4RG detectors. In the sequential design architecture, the imager further serves as a set of pre-optics for the IFS. The imager subsystem also hosts a cold stop, ADC, and a suite of filters. The imager relays the science light to the IFS via pickoff mirrors which are located just prior to the imager detectors. The Lenslet pickoff mirror is stationary. The Slicer pickoff is deployable and is able to move in-and-out of the optical path, in front of the fixed Lenslet pickoff. The current baseline design, presented herein, employs a set of reflective optics. Several refractive and reflective optical designs were investigated, and the IRIS team has selected the highest throughput optical design for the imager. The requirement of IRIS imager is summarized in Table 1.

\begin{tabular}{|l|l|}
\hline Items & Requirements \\
\hline Wavelength range & $0.84-2.4 \mu \mathrm{m}$ \\
\hline Field of view & Larger than 16 " x 16" \\
\hline Detector & H4RG-10 (10 microns pitch) \\
\hline Configuration & Collimator + Camera \\
\hline F/\# of camera & $17.19+/-0.86$ \\
\hline Wavefront error & WFE shall be less than $40 \mathrm{~nm}$ after $60 \mathrm{x} 60$ order correction by NFIRAOS DMs. \\
\hline Throughput & Greater than 45\% with Y, Z, J, H, K, Ks broadband filters. \\
\hline Pupil image & $\begin{array}{l}\text { The collimator optics shall form a real image of the telescope pupil in the } \\
\text { collimated beam. }\end{array}$ \\
\hline Pupil aberration & $\begin{array}{l}\text { Geometrical spot diameter shall be less than } 0.4 \% \text { of the diameter of the pupil } \\
\text { image. }\end{array}$ \\
\hline Beam transfer to IFS & $\begin{array}{l}\text { Imager shall transfer science light beam to the lenslet and slicer IFS optics via } \\
\text { pickoff mirrors located in front of the imager detectors. }\end{array}$ \\
\hline
\end{tabular}

Table 1. Top level requirement of IRIS imager.

The Imager optics are based on a conventional collimator- camera configuration and locates both the ADC and filter suite within the collimated beam. The light from NFIRAOS enters IRIS as an F/15 beam at the NFIRAOS focal surface, which is $200 \mathrm{~mm}$ above the IRIS cryostat entrance window. The light is collimated by three conic mirrors (TMA : three mirror anastigmat) in the collimator assembly and refocused onto the four Hawaii-4RG detectors with an F/17.2 beam by three additional conic mirrors, which form the camera 
assembly. For packaging, the design provides a collimated beam with a length of $1,850 \mathrm{~mm}$ for the cold stop, filters, ADC, and fold mirrors. The collimator assembly also produces a pupil image (with a $95 \mathrm{~mm}$ diameter) enabling background suppression by the cold stop. The detail of optical design and performance analysis are summarized in the paper in $2016^{3}$.

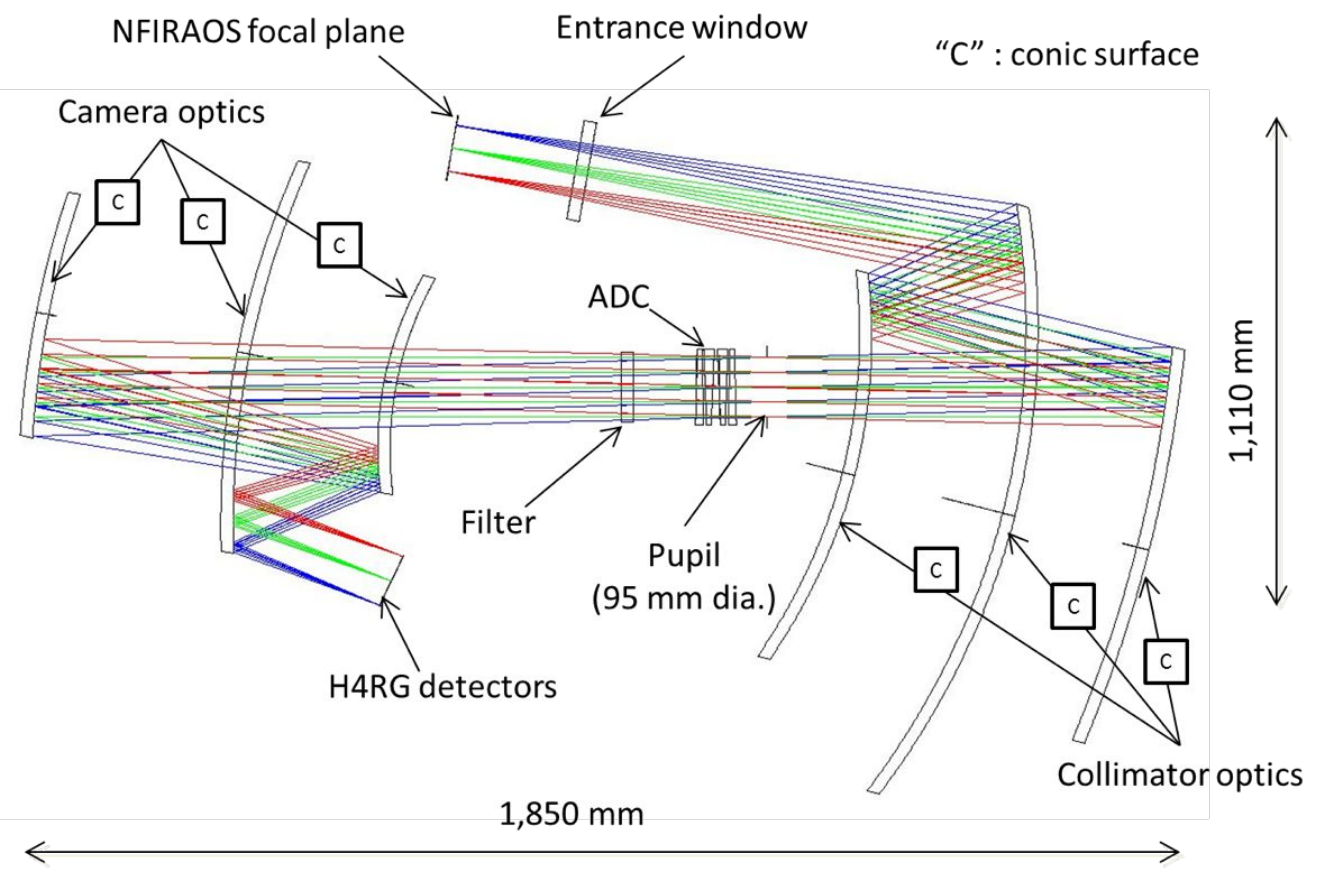

Figure 1. Optical design of IRIS imager.

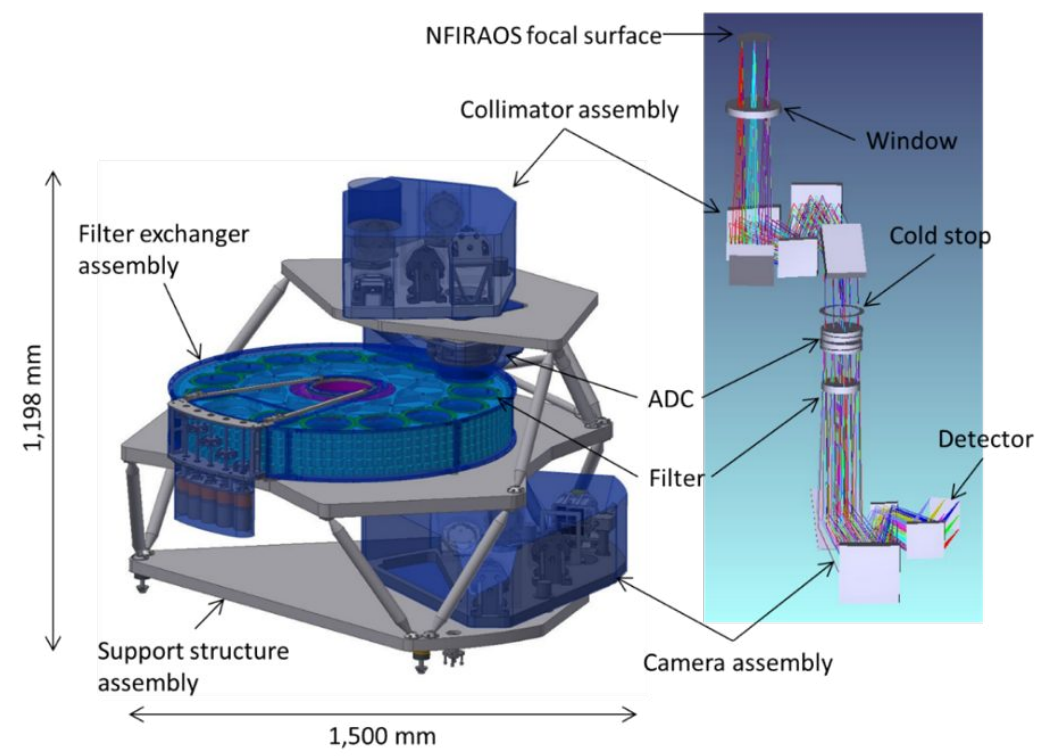

Figure 2. Mechanical model (left) and optical model (right) of IRIS imager.

Figure 2 highlights the latest mechanical design while further depicting the imager's optical path. The imager optics are based on a conventional collimator - camera configuration and locates both the ADC and filter 
suite within the collimated beam. Each assembly is individually supported by one of the three floor plates, which are in turn interconnected to form the imager support structure.

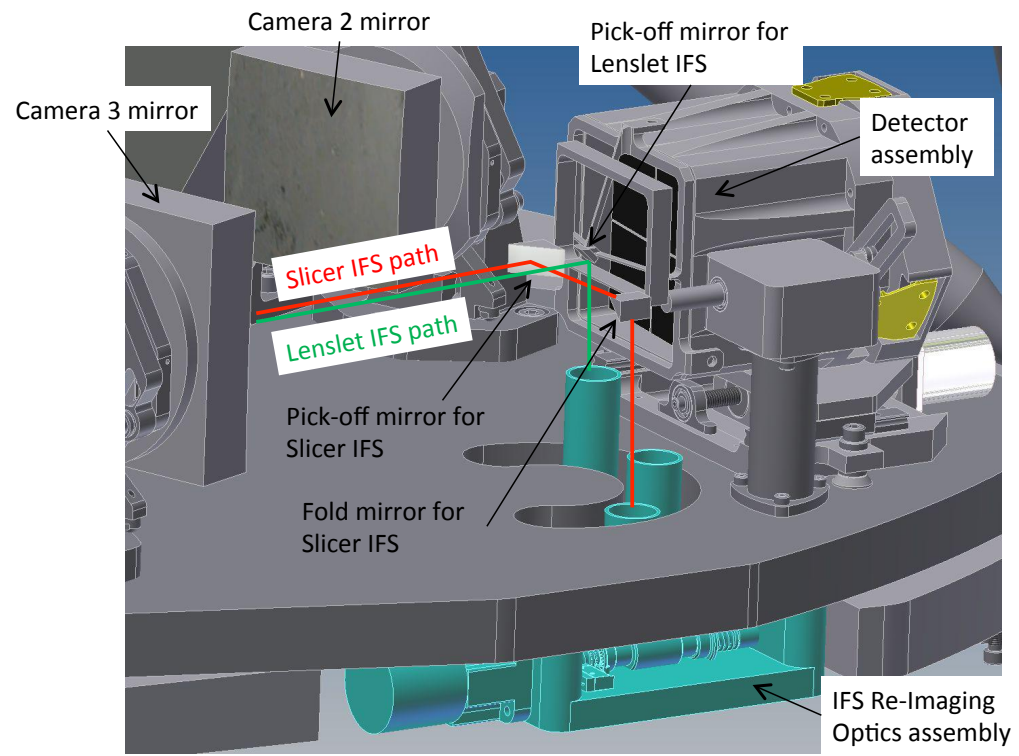

Figure 3. Close-up view of the area around the imager detector assembly.

Figure 3 provides a close-up view of the camera assembly showing both pick-off mirrors in front of the imager detectors. The optical path showing the orientation of the relayed beam is shown for each IFS channel. The lenslet IFS pick-off mirror is fixed to the detector assembly, while the slicer IFS pick-off mirror moves in-and-out, thus serving to switch between the two IFS modes. The imager subsystem occupies a space envelop of 1,500 mm diameter and $1198.2 \mathrm{~mm}$ length and extends to the upper part of the science cryostat, and has a total mass of $536.9 \mathrm{~kg}$. Due to stringent requirements on imaging performance, extra care was exercised in the selection of imager materials to minimize differential thermal contraction between optics and mechanical housing in the cryogenic environment.

\section{MECHANICAL DESIGN}

An exploded CAD model view of the Imager mechanical design is shown in Figure 4.

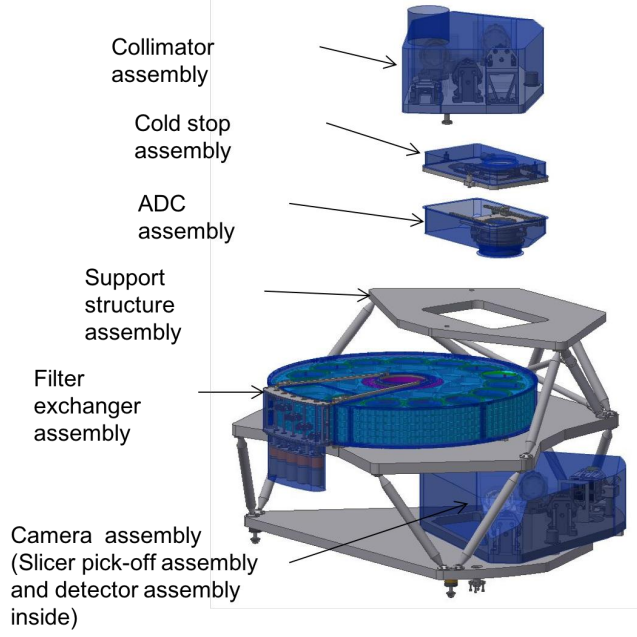

Figure 4. Exploded CAD model view of the imager mechanical design. 
The imager mechanics consists of collimator, cold stop, ADC, filter exchanger, camera, and detector assemblies as well as the slicer IFS pick-off mirror and support structure assemblies. The light path is surrounded by a baffle mechanism, which is shown in blue in Figure 4, which provides a light-tight environment using tongue and groove and step features, which are painted black on the mating surfaces. The collimator assembly and camera assembly hold the mirrors accurately and rigidly using bonding pads and flexure mechanisms. Thermal contraction of the base plate of collimator and camera assembly at the cryogenic temperature around $77 \mathrm{~K}$ cooled by the liquid nitrogen is handled by the fixed point on the optical axis under the first flip mirror (see Figure 5 for collimator assembly). Two other mechanical anchors can be compensate the thermal contraction in the direction towards the fixed point. The base plate of collimator and camera assemblies will be made by the stainless invar, whose main components are $\mathrm{Cr}$ and $\mathrm{Co}$ in the invar steal material instead of Ni. The same invar material is used for bonding pads. The cold stop and ADC assemblies support both rotary and linear motion to track field rotation to enable observations with and without the cold stop and ADC, respectively. The filter exchanger assembly is equipped with five filter wheels to accommodate 70 filters (See Figure 6). The slicer IFS pickoff mirror assembly moves in-and-out via a rotary motion to switch between IFS observing modes. The detector assembly secures the four detectors, provides positional adjustment features, supports the lenslet IFS pickoff mirror, and provides the required thermal and electrical insulation. The support structure assembly provides a three-story platform to which all other assemblies mount.

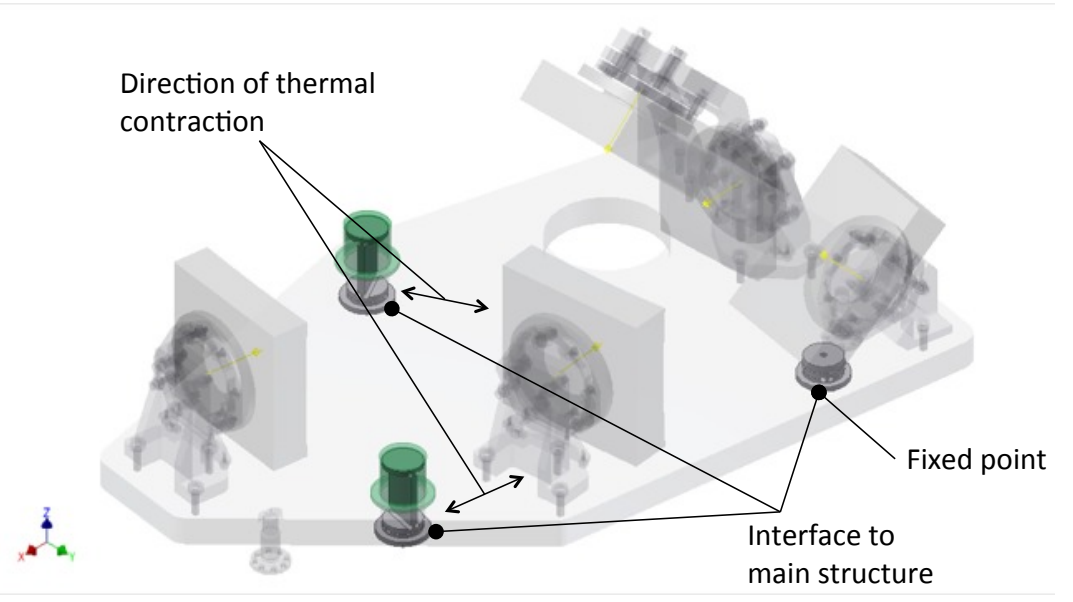

Figure 5. CAD model of collimator assembly.

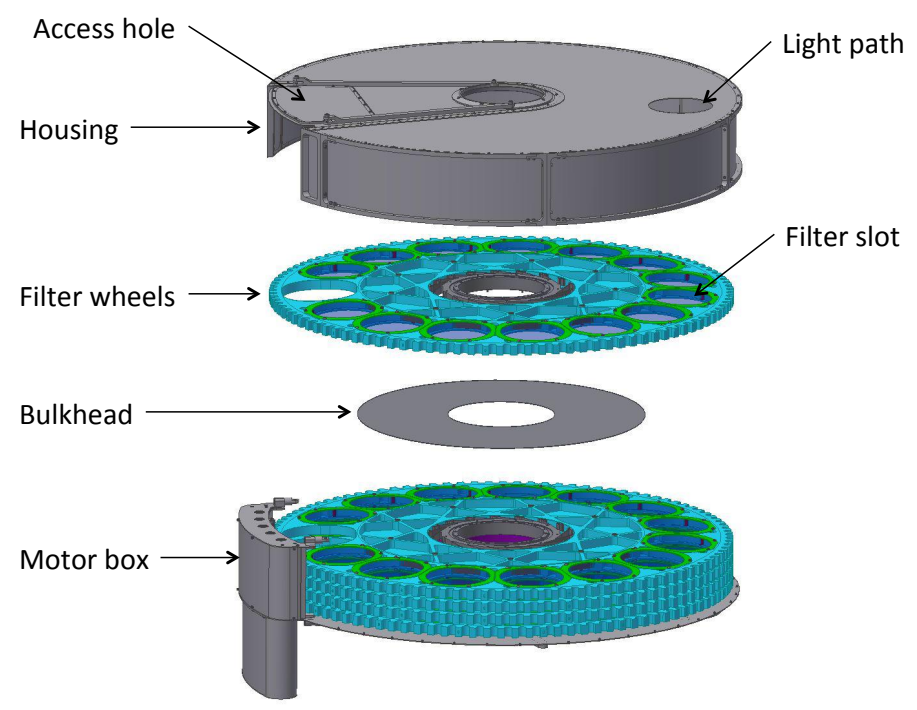

Figure 6. Exploded CAD model of filter wheel assembly. 


\section{PROTOTYPINNG}

The NAOJ IRIS imager team have prototyped assemblies, components, and elements for which they recognized risks. Table 2 lists the prototyped items that the Imager team have conducted so far. The prototyping of cold stop stage are reported in the previous conference of Astronomical Telescopes and Instrumentation in $2016^{4}$. The following subsections provide brief report of this prototyping work.

\begin{tabular}{|l|l|}
\hline \multicolumn{1}{|c|}{ Prototyped items } & \multicolumn{1}{c|}{ Purpose } \\
\hline Cold stop stage & $\begin{array}{l}\text { I) For verifying functionalities of a rotary and linear stage at } \\
\text { cryogenic operating temperatures } \\
\text { II) To show compliance to performance requirements }\end{array}$ \\
\hline Hall effect sensors & For characterization at the operating temperature \\
\hline Rotary bearing, ball screw, and linear slide & For lifetime durability tests at the operating temperature \\
\hline $\begin{array}{l}\text { Full size off axis concave ellipsoid with } \\
1 / 20 \text { quality }\end{array}$ & For checking manufacturability and verification methods \\
\hline $\begin{array}{l}\text { Full size off axis convex hyperboloid with } \\
1 / 20 \text { quality }\end{array}$ & For checking manufacturability and verification methods \\
\hline Bonding pads for the mirror mount & $\begin{array}{l}\text { I) For strength tests at the operating temperature } \\
\text { II) For characterization at the operating temperature } \\
\text { III) For checking effects on wavefront error at the operating } \\
\text { temperature }\end{array}$ \\
\hline $\begin{array}{l}\text { Reflective coating with enhanced silver } \\
\text { and multi-layer dielectric coatings. }\end{array}$ & $\begin{array}{l}\text { I) Reflectivity measurement } \\
\text { II) For checking effects on wavefront error at the } \\
\text { operating temperature }\end{array}$ \\
\hline
\end{tabular}

Table 2. List of prototyped items and purposes.

\subsection{Hall effect sensors}

The Imager requires opt-mechanical stages which are operable at vacuum and cryogenic temperature. To define the reference position of the stages in such conditions, switch-like devices are required. An investigation was carried out to find such devices, and one such candidate that was found was a combination of a hall element and magnet. This combination was used for position determination of the stages in MOIRCS and HiCIAO at Subaru Telescope, and has been in operation for more than ten years. SmCo magnets were selected because their magnetic fields between $77 \mathrm{~K}$ to room temperature remain relatively constant. We found that hall sensor, HG 106A, manufactured by Asahi Kasei Microdevices (AKM), showed the adequate output signal at $77 \mathrm{~K}$. The purpose of the test is to determine the characteristics of hall elements and magnets as well as to establish an in-house implementation technique for the reference position sensor.

\subsection{Lifetime test}

IRIS is required to operate for 23 years with a preventive maintenance period of 3 months between 13 and 10 years of consecutive operations. Operating a 'motion mechanism' at cryogenic temperatures for 10 years without maintenance is nontrivial. Therefore, we conducted durability tests for some of the key elements; rotary bearing, ball screws and linear slide, to provide the basis for illustrating compliance towards the lifetime requirements. The durability tests were conducted at $50 \mathrm{~K}$ using a test cryostat available at NAOJ. We defined the lifetime operation requirements for each element as (1) 2,160,000 degrees for rotary motion, and (2) 4,500,000 mm for linear motion, which were based on past operational results for instruments at Subaru Telescope. It was demonstrated that the rotary bearing with ceramic balls, steel crown separators, and the ball screw with the MoST coating survived the lifetime operations.

\subsection{Bonding test}

We chose bonding pads and flexure springs for mounting the mirror substrate to the mirror holder. Stainless Invar, which has similar characteristics of thermal expansion with fused silica, was selected as the bond pad material and was done so to minimize differential thermal contraction with the mirror substrate, which itself is made from fused silica. Armstrong A-12 was selected for the adhesive as it has been well characterized even at low temperatures and has heritage in the use 
in previous astronomical instruments. One of the concerns of using adhesives for structural elements is its strength. We have conducted strength tests at $77 \mathrm{~K}$ with test pieces of the mirror substrate, bonding pad, and the adhesive.

The bonding surface of fused silica are treated in three different way. One is polished, others are polished by 600 grit and 320 grit $\mathrm{SiC}$ abrasive paper. The thickness of adhesive is controlled by glass beads, whose diameter is $0.07 \mathrm{~mm}$. We applied the force both in share direction and tensile direction at $77 \mathrm{~K}$ and measured the force when it break up, by monitoring the acceleration on the substrate. Figure 7 shows the Weibull plot for each conditions.

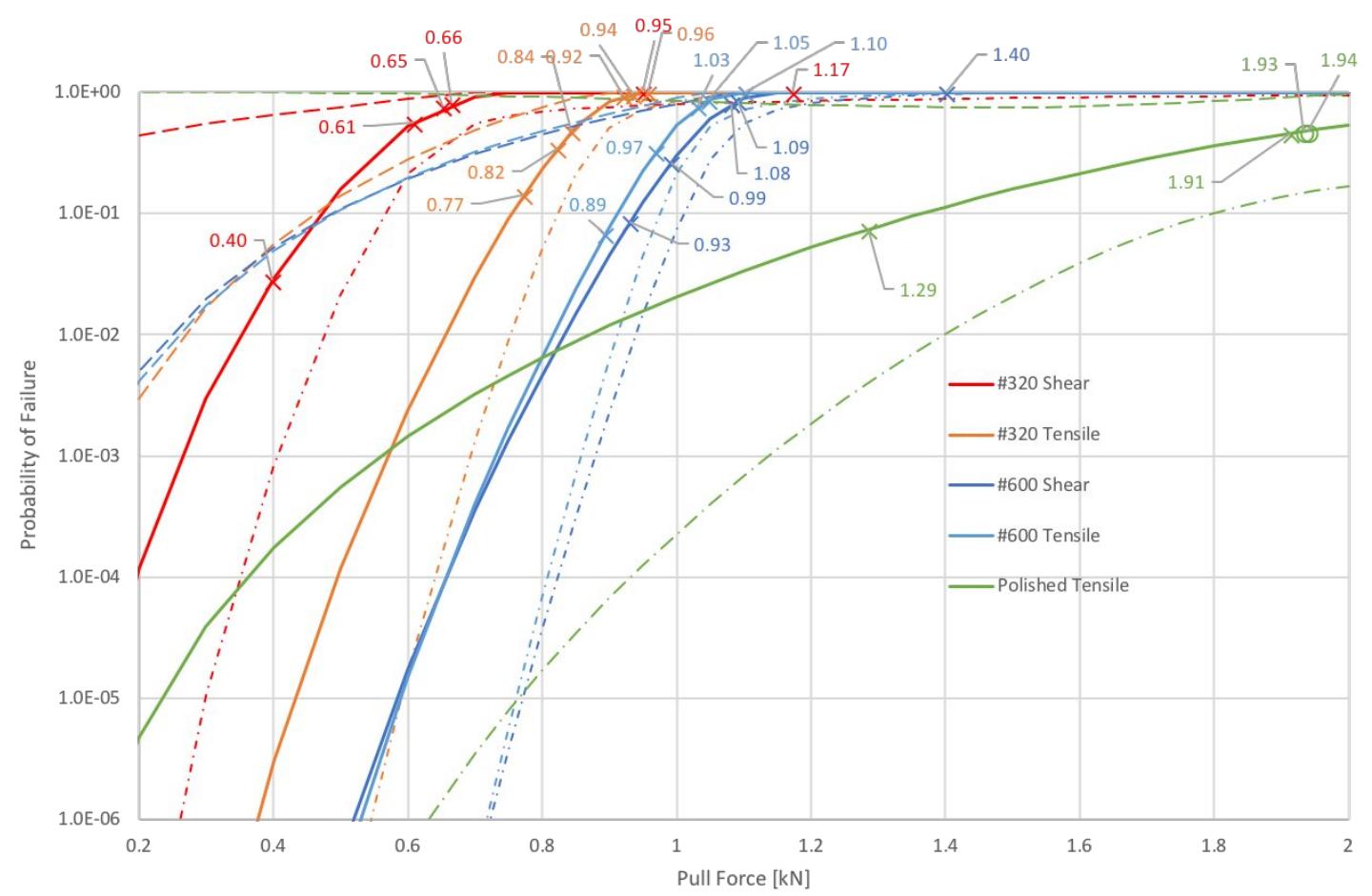

Figure 7. Weibull plot, indicates the probability of failure as a function of pulling force for each condition of bonding.

Bonding on the polished surface has larger strength, but the threshold of breaking pull forces are scattered. Breaking pull force is smaller but predictable for a bonding on the surface scratched with \#600 grinder. The breaking pull force is minimum, when we use 320 grit $\mathrm{SiC}$ abrasive paper.

\section{CONCLUSION}

The optical and mechanical design of IRIS imager and prototyping activities conducted during the preliminary design phase are summarized.

\section{REFERENCES}

[1] Larkin, J. E., Moore, A. M., Wright, S. A., Wincentsen, J. E., Anderson, D., Chisholm, E. M., Dekany, R. G., Dunn, J. S., Ellerbroek, B. L., Hayano, Y., Phillips, A. C., Simard, L., Smith, R., Suzuki, R., Weber, R. W., Weiss, J. L. and Zhang, K., "The Infrared Imaging Spectrograph (IRIS) for TMT: instrument overview," Proceedings of the SPIE, Volume 9908, id. 99081W 13 pp. (2016)

[2] Herriot, G., Andersen, D., Atwood, J., Boyer, C., Byrnes, P., Caputa, K., Ellerbroek, B., Gilles, L., Hill, A., Ljusic, Z., Pazder, J., Rosensteiner, M., Smith, M., Spano, P., Szeto, K., Véran, J.-P., Wevers, I., Wang, L. and Wooff, R., "NFIRAOS: first facility AO system for the Thirty Meter Telescope", Proceedings of the SPIE, Volume 9148, id. 91481011 pp. (2014). 
[3] Tsuzuki, T., Suzuki, R., Harakawa, H., Ikenoue, B., Larkin, J., Moore, A., Obuchi, Y., Phillips, A. C., Saito, S., Uraguchi, F., Wincentsen, J., Wright, S. and Hayano, Y., "The Infrared Imaging Spectrograph (IRIS) for TMT: optical design of IRIS imager with "co-axis double TMA"," Proceedings of the SPIE, Volume 9908, id. 9908AE 18 pp. (2016).

[4] Uraguchi, F., Obuchi, Y., Ikenoue, B., Saito, S., Suzuki, R. and Hayano, Y., "The Infrared Imaging Spectrograph (IRIS) for TMT: prototyping of cryogenic compatible stage for the imager", Proceedings of the SPIE, Volume 9908, id. 9908A8 10 pp. (2016). 